\title{
Hemmbarkeit proteolytischer Enzyme in pathologischen Nasensekreten und von Leukocytenproteasen durch den natürlichen Proteaseninhibitor des Nasensekrets ${ }^{1)}$
}

\author{
Von K. Hochstrasser, R. Reichert, Monika Matzner und E. Werle, \\ unter Mitarbeit von Sibylle Schwarz \\ Biocbemisches Laboratorium (Leitung: Priv. Doz. Dr. K. Hocbstraßer) der HNO-Klinik \\ (Direktor: Prof. Dr. H. H. Naumann) \\ Institut für Kliniscbe Chemie und Kliniscbe Biocbemie \\ (Direktor: Prof. Dr. Dr. E. Werle) der Universität München
}

(Eingegangen am 30. Juli 1971)

\begin{abstract}
Menschliches Nasensekret enthält physiologischerweise einen Proteaseninhibitor, der neben Trypsin, Chymotrypsin und Pronase auch leukocytäre Proteasen zu hemmen vermag und nicht mit Inhibitoren des Blutserums identisch ist. Unter pathologischen Bedingungen erscheint im menschlichen Nasensekret proteolytische Aktivität, die in gleicher Weise wie Leukocytenproteasen von Inhibitor aus normalem Nasensekret hemmbar ist. Dem Inhibitor kann eine Schutzfunktion für die Nasenschleimhaut gegen Proteasen aus zerfallenden Leukocyten zugesprochen werden. Der Abfall des Inhibitorspiegels im Nasensekret bei verschiedenen Rhinopathien kann auf die Wechselwirkung zwischèn Inhibitor und Leukocytenproteasen oder noch nicht näher identifizierter Proteasen zurückgeführt werden.
\end{abstract}

\section{The inbibition of proteolytic enzymes in pathological nasal secretion and of leucocyte proteases by the natural protease inbibitor of nasal secretion}

Under physiological conditions human nasal mucus contains a proteinase inhibitor which inhibits trypsin, chymotrypsin, pronase, and leucocytic proteases. The inhibitor is not identical with inhibitors from blood serum. In pathologiçal conditions proteolytic activity appears in human nasal mucus. This activity, like that of leucocytic proteases, is blocked by the inhibitor from normal nasal mucus. We propose that the inhibitor protects the nasal mucus membrane against proteases from decomposed leucocytes. The dectease of the inhibitor level in nasal mucus in different rhinopathia may be explained as an interaction of inhibitor and leucocytic proteases or other unidentified proteases.

Menschliches Nasensekret enthält unter physiologischen Bedingungen einen Hemmstoff, der die Proteasen $^{2}$ ) Trypsin, Chymotrypsin und die Pilzprotease Pronase (aus Streptomyces griseus) inhibieren kann. Die spezifischen Proteasen Plasmin und Kallikrein werden nicht gehemmt. Das Inhibitormolekül ist so gebaut, $\mathrm{da} ß$ es je nach angebotenem Enzym entweder $1 \mathrm{Mol}$ Trypsin oder $2 \mathrm{Mol}$ Chymotrypsin unter Inaktivierung bindet. Nach vorheriger Absättigung mit Trypsin kann noch $1 \mathrm{Mol}$ Chymotrypsin gebunden werden, nach Absättigung mit Chymotrypsin ist die inhibitorische Wirkung gegen Trypsin blockiert (1). Der Hemmstoff besitzt ein Molekulargewicht von etwa 13000. Er ist nicht mit Inhibitoren des Blutserums identisch, so daß als Bildungsort die Nasenschleimhaut selbst angesehen werden kann. Die Konzentration des Inhibitors im Nasensekret - bezogen auf Trypsinhemmung und sezerniertes Protein - liegt in gleicher Größenordnung wie die Proteasen-Hemmstoffkonzentrationen im Serum.

1) Die Arbeit wurde mit Mitteln des SFB 51 der deutschen Forschungsgemeinschaft gefördert.

2) Enzyme: Trypsin (EC 3.4.4.4), Chymotrypsin (EC 3.4.4), Pronase (EC 3.4.), Plasmin (EC 3.4.4.14), Kallikrein (EC 3.4.4.21).
Bei Untersuchungen, die Einblick in die physiologische Funktion dieses Inhibitors geben sollten, konnten wir bei bestimmten Rhinopathien eine deutliche Verminderung des Inhibitorspiegels im Sekret beobachten (2). Zur Erklärung dieser Befunde konnte man eine verminderte sekretorische Leistung und verminderte Funktionsfähigkeit der Nasenschleimhaut annehmen. Jedoch ist wegen der biochemischen Wirkung des Inhibitors noch eine andere Deutung möglich. Der Ablauf einer Entzündung ist unter anderem dadurch gekennzeichnet, daß es im entzündeten Gewebe zur Aktivierung von Gewebsproteasen kommt, die ihrerseits das entzündungsverstärkende Kallikrein-Kinin-System aktivieren (3). Bei bakteriell ausgelösten Entzündungen ist die Aktivierung dieses Systems auch durch Bakterienproteasen oder durch proteolytische. Enzyme zerfallender Leukocyten denkbar. Nimmt man diesen Reaktionsmechanismus an, so kann dem Proteaseninhibitor im Nasensekret eine Schutzfunktion zugesprochen werden, sofern er solche Proteasen zu hemmen vermag. Die Nasenschleimhaut wärè somit vor proteolytischem Angriff und den daraus resultierenden Folgen geschützt. Dieser Schutzmecharismus ist jedoch an eine ausreichende Inhibitorproduktion gebunden. Das Absinken des Inhibitorspiegels könnte bei ent- 
zündlichen Rhinopathien als Verbrauchsreaktion angesehen werden: Der sezernierte Inhibitor reagiert mit den beteiligten Proteasen unter Komplexbildung. In unserem Untersuchungsmaterial fanden sich Sekrete, in denen praktisch kein Inhibitor mehr nachweisbar war. Beim Zutreffen der dargestellten Überlegungen war in diesen Sekreten bei offensichtlicher Überforderung des inhibierenden Systems proteolytische Aktivität zu erwarten.

\section{Methoden und Material}

Sekretgewinnung

Nasensekret wurde durch Einlegen mit physiol. NaCl-Lösung getränkter Baumwolltampons in die Nasengänge gewonnen. Das

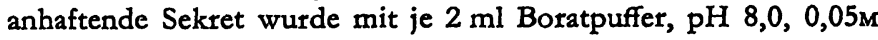
extrahiert.

\section{Leukocytenproteasen}

Zur Gewinnung von Leukocytenproteasen wurde Eiter aus Kieferhöhlen verschiedener Patienten verwendet. Das Material wurde im Zellhomogenisator in 0,05M Boratpuffer, $\mathrm{pH} 8,0$, aufgeschlossen, hochtourig zentrifugiert und der klare Uberstand für die Untersuchungen verwendet.

\section{Substrate}

$\mathrm{N}^{\alpha}$-Benzoyl-DL-arginin-4-nitroanilid-hydrochlorid, Merck; Succinyl-L-phenyl-alanin- $p$-nitroanilid, Boehringer; Azocasein, Serva.

\section{Inhibitoren}

Sekretinhibitor angereichert nach (1); Inhibitor aus Submandibularisdrüsen des Hundes (4); Inhibitor aus Aracbis bypogaea (5), Inhibitor aus Phaseolus vulgaris, Laborpräparat; Trypsin-Kallikreininhibitor aus Rinderorganen $=$ Trasylol, Bayer; $\alpha_{1}$-Antitrypsin in Form von Serum.

\section{Prüfung auf proteolytische Aktivität}

a) Zur Prüfung auf trypsinäbnliche Aktivität im Nasensekret wurde untersucht, inwieweit das Substrat $\mathrm{N}^{\alpha}$-Benzoylarginin- $p$-nitroanilid hydrolysiert wird (6).

b) Auf chymotryptische Aktivität wurde analog mit Succinylphenylalanin-p-nitroanilid als Substrat geprüft (6).

c) Nach unspezifischen Proteasen wurde mit Azokasein als Substrat gesucht (10). Bei der Spaltung dieses Substrats entstehen lösliche, nicht fällbare, gelbgefärbte Spaltprodukte. Eine Angabe über definierte Enzymeinheiten ist in diesem Fall wegen der Unspezifität des Substrates nicht möglich. Wir vergleichen daher die proteolytische Aktivität des Nasensekrets mit der Wirkung von definierten Trypsinmengen auf das Substrat. Wir geben an, daß ein Nasensekret gegen Azokasein eine proteolytische Aktivität wie $x \mathrm{mU}$ Trypsin entfaltet. Die zur wirkungsgleichen Trypsinmenge identische Aktivität wird aus einer Eichkurve ermittelt. Die proteolytische Aktivität des Nasensekrets wurde bei $\mathrm{pH} 8,0$ (0,1 M Phosphat) bestimmt. Ausführung: $0,2 \mathrm{ml}$ Sekret werden mit $0,8 \mathrm{ml}$ Phosphat-Puffer, $\mathrm{pH} 8,0,0,1 \mathrm{M}$ vermischt; $2 \mathrm{ml} \mathrm{2proz}$. Azokaseinlösung im gleichen Puffer, 60 Min. bei $37^{\circ}$; Fällung mit $3 \mathrm{ml} 5$ proz. Trichloressigsäure. Nach Zentrifugieren wird der Uberstand filtriert und gegen den Reagenzienleerwert bei $366 \mathrm{~nm}$ gemessen. Eichkurven mit definierten Trypsinmengen bei gleichem Substrat und unter gleichen Bedingungen wẹden analog aufgestellt.

d) Die proteolytische Aktivität der Leukocytenhomogenate wurde mit der gleichen Methodik bestimmt. Ebenso wurde die pH-Abhängigkeit der Leukocytenproteasen untersucht. Es wurden nur jeweils Substrat und Leukocytenkonzentrat im entsprechenden KNa-Phosphatpuffer gelöst.

Hemmversuche

Die Hemmbarkeit der proteolytischen Wirkung der Nasensekrete und der Leukocytenhomogenate wird mit den Versuchsanord- nungen c) und d) untersucht. Den Inkubationsansätzen werden steigende Mengen Inhibitor, ausgedrückt in Trypsininhibitormilliunits, zugesetzt.

\section{Ergebnisse}

Wir wählten zur Untersuchung der vorliegenden Fragestellung eine Gruppe von Patienten mit verschiedenen Rhinopathien aus, deren Nasensekrete normalen oder stark erniedrigten Inhibitorgehalt aufwiesen (Tab. 1). Aufgrund des bisher bekannten Hemmspektrums des Inhibitors - Hemmung von Trypsin und Chymotrypsin - untersuchten wir diese Sekrete auf trypsin- und chymotrypsinähnliche $\mathrm{Ak}$ tivität. Wir konnten in keinem Fall Spaltung der für beide Enzyme spezifischen Substrate wie $\mathrm{N}^{\alpha}$-Benzoylarginin- $p$-nitroanilid und Succinylphenylalanin- $p$-nitroanilid nachweisen. Bei der Verwendung von Azokasein als unspezifisches Proteasensubstrat fanden wir in einigen Fällen eine erhebliche, proteolytische Aktivität im Nasensekret (Tab. 1).

Tab. 1

Inhibitorgehalt und proteolytische Aktivität in normalen und pathologischen Nasensekreten

\begin{tabular}{rccl}
\hline Fall-Nr. & $\begin{array}{c}\text { ImU*/mg } \\
\text { Protein }\end{array}$ & $\begin{array}{c}\text { Proteolyt. } \\
\text { Aktivität** }\end{array}$ & \multicolumn{1}{c}{ Befund } \\
\hline 7 & 11,4 & 0 & $\begin{array}{l}\text { hypertrophierende } \\
\text { Sinusitis }\end{array}$ \\
13 & 31,6 & 0 & ohne B. \\
17 & 4,0 & 0,02 & Sinusitis \\
19 & 0,7 & 0,7 & eitriges Sekret \\
10 & 9,4 & 0 & ohne B. \\
11 & 0,4 & 2,9 & eitrige Sinusitis \\
4 & 0,1 & 1,1 & Pansinusitis \\
6 & 11,7 & 0 & ohne B. \\
5 & 11,8 & 0 & ohne B. \\
\hline
\end{tabular}

* Inhibitormilliunits

* Proteolytische Aktivität wirkungsgleich mit mU Trypsin/mg Protein

Falls ein Zusammenhang zwischen Proteaseninhibitor im Nasensekret unter physiologischen Bedingungen und aktiver Protease im pathologischen Zustand besteht, so ist zu erwarten, daß die Protease des pathologischen Nasensekrets durch isolierten Sekretinhibitor hemmbar ist. In der Tat konnten wir feststellen, daß die proteolytische Wirkung pathologischer Nasensekrete durch Inhibitor aus normalem Nasensekret hemmbar ist. Mit Sekretinhibitor sind jedoch nur etwa $70 \%$ der proteolytischen Aktivität der pathologischen Nasensekrete hemmbar, wie aus der Hemmkurve (Abb. 1, Kurve 1) $\mathrm{zu}$ entnehmen ist. Die restliche, nicht hemmbare Aktivität dürfte von Exopeptidasen ausgehen. Der Typ der Hemmkurve entspricht völlig den Verhältnissen, die in unserem Arbeitskreis bei anderen Proteasen und Inhibitoren gefunden wurden (4).

Die von uns untersuchten pathologischen Nasensekrete mit proteolytischer Wirkung enthielten jeweils Leukocyten. Wir vermuteten deshalb, daß die proteolytische Wirkung dieser Sekrete durch Leukocytenproteasen hervorgerufen wird. Sollte diese Annahme zutreffen, so war Hemmbarkeit von Leukocytenproteasen durch Sekretinhibitor zu erwarten. In Abbildung 1, Kurve 2 


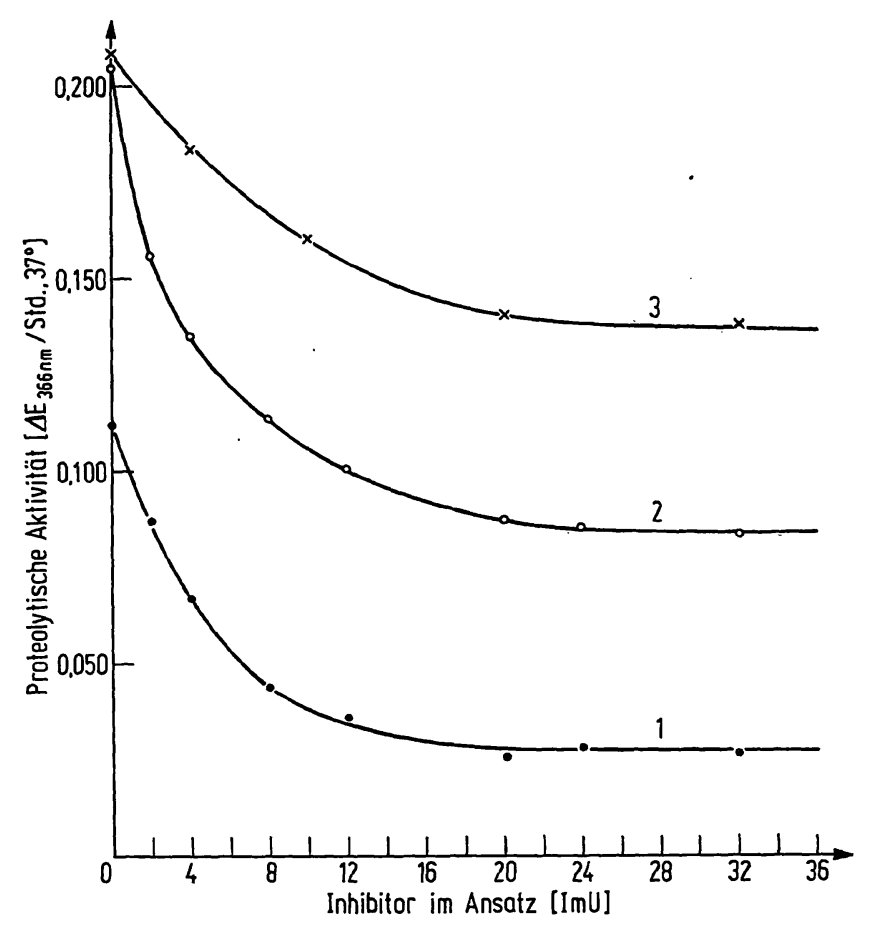

Abb. 1

Hemmung der proteolytischen Wirkung von pathologischem Nasensekret (1) und von Leukocytenproteasen durch steigende Mengen von Nasensekretinhibitor (2) bzw. Blutserum (3)
I $\mathrm{mU}=$ Inhibitormilliunits gegen Trypsin

Ansätze: $0,2 \mathrm{ml}$ Sekret oder Leukocytenhomogenat (=16 mg Trockensubstanz), Inhibitorlösung auf $1 \mathrm{ml}$ mit Phosphatpuffer, $\mathrm{pH} 8,0$ $0,1 \mathrm{M}$, auffüllen; $2 \mathrm{ml}$ 2proz. Azokaseinlösung in gleichem Puffer $60 \mathrm{Min}$. bei $37^{\circ} ; 3 \mathrm{ml} 5 \mathrm{proz}$. Trichloressigsäure. Im Überstand Extinktion gegen Reagenzienleerwert bei $366 \mathrm{~nm}$ gemessen

Erzielbarer Endhemmgrad $1=75 \%, 2=56 \%, 3=30 \%$ Hemmung

ist eine Hemmkurve dargestellt, die mit Leukocytenhomogenat und Sekretinhibitor erhalten wurde. Die Hemmung der Leukocytenproteasen verläuft völlig

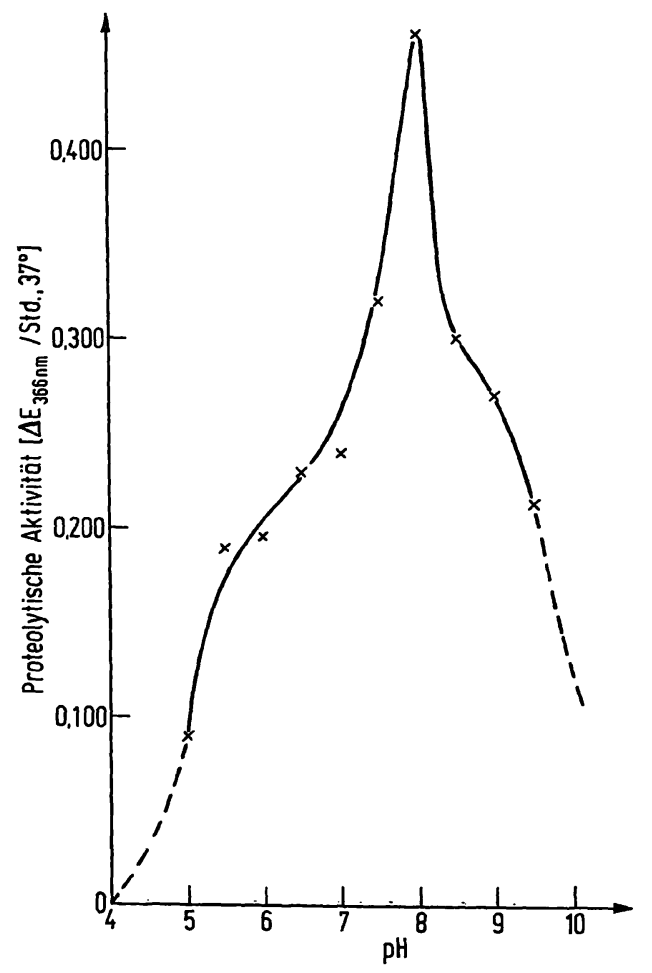

Abb. 2

Aktivität der Leukocytenproteasen bei verschiedenen pH-Werten. Ausführung wie in Legende zu Abbildung 1. KNa-Phosphatpuffer,

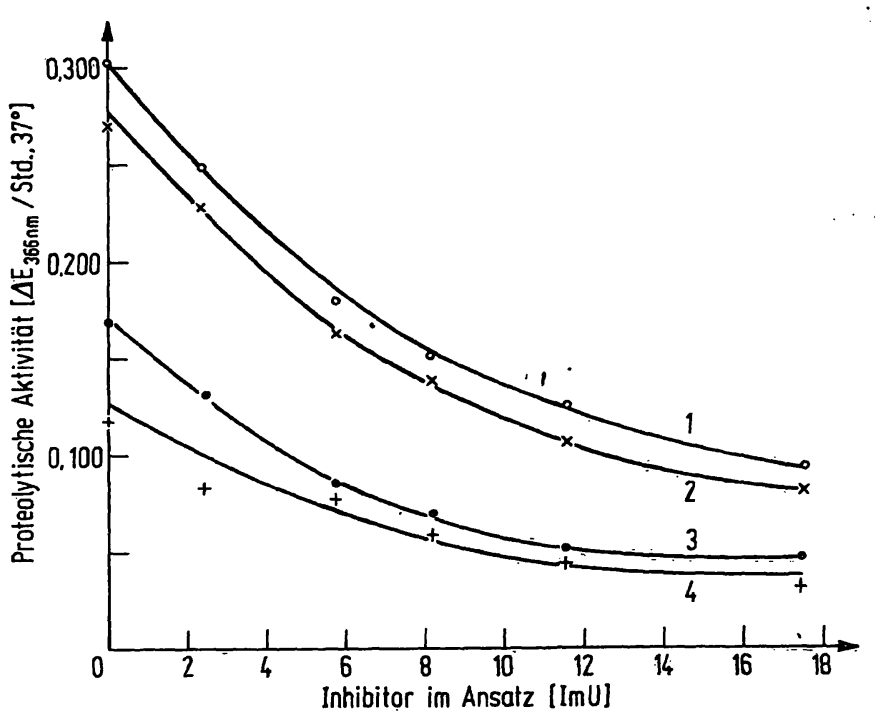

Abb. 3

Hemmbarkeit von Leukocytenproteasen bei verschiedenen pH-Werten, $1=\mathrm{pH} 8,2=\mathrm{pH} 7,3=\mathrm{pH}_{\text {Nasensekret }} 4,4=\mathrm{pH} 5$, durch Inhibitor aus Ausführung wie in Legende zu Abbildung 1. KNa-Phosphațuffer, $0,1 \mathrm{M}$

parallel der Hemmung von proteolytisch wirkendem Nasensekret.

Die proteolytische Wirkung von Leukocytenhomogenaten ist im weiten Bereich von $\mathrm{pH} 5$ bis 8,5 mit einem deutlichen Maximum bei $\mathrm{pH}$ 8,0 nachweisbar (Abb. 2). Hemmung dieser proteolytischen Aktivität durch Sekretinhibitor ist im ganzen $\mathrm{pH}-$ Bereich möglich. Die maximal erzielte Hemmung liegt jeweils in der Größenordnung von etwa $70 \%$ (Abb. 3).

Die Proteaseninhibitoren aus Hundesubmandibularisdrüsen, Rinderorganen, Phaseolus vulgaris und Arachis bypogaea vermochten Leukocytenproteasen nicht $\mathrm{zu}$ hemmen. Humanplasma hemmte die Leukocytenproteasen wie der Sekretinhibitor, jedoch lag die maximale Hemmung exheblich niedriger (Abb. 1, Kurve 3) als $70 \%$. Wie Tabelle 1 belegt, ist proteolytische Aktivität im Sekret nur dann nachweisbar, wenn der Inhibitor im Sekret weitgehend fehlt. Bei noch nachweisbarem Inhibitor ist in keinem Fall proteolytische Aktivität im Nasensekret zu beobachten.

\section{Diskussion}

Der Proteaseninhibitor des menșchlichen Nasensekrets vermag neben Trypsin und Chymotrypsin Proteasen aus zerfallenen Leukocyten zu hemmen. Wir sehen darin die wichtigste physiologische Funktion des Inhibitors. Diese Annahme ist jedoch erst nach Diskussion der sich scheinbar widersprechenden Befunde gerechtfertigt. Einmal wurde festgestellt, daß in Nasensekreten, in denen noch nachweisbare Inhibitormengen vorhanden sind, in keinem Fall proteolytische Aktivität zu finden war. Zum zweiten vermag aber angereicherter Inhibitor jeweils nur etwa 70\% der Aktivität proteolytisch wirkender Nasensekrete oder von Leukocytenhomogenaten zu hemmen. Diese Befunde scheinen nur teilweise die Annahme zu rechtfertigen, daß die physiologische Funktion des Inhibitors darin besteht, 
Proteasen aus zerfallenden Leukocyten $\mathrm{zu}$ inhibieren. Die verwendeten Enzymquellen, pathologisches Nasensekret und Eiter sind jedoch Produkte pathophysiologischer Vorgänge, und es ist damit zu rechnen, daß in diesen Sekreten neben leukocytären Proteasen auch bakterielle Proteasen und Exopeptidasen vorkommen. Auch muß man an aktivierte Gewebsproteasen denken. $\mathrm{Da}$ wir zeigen konnten, daß Bakterienproteasen und Exopeptidasen von unserem Sekretinhibitor nicht gehemmt werden (1), sind somit die verbleibenden, nicht hemmbaren Restaktivitäten in den untersuchten Sekreten erklärbar (siehe dazu auch (4)).

Der Abfall des Inhibitorspiegels im Verlauf einer Rhinopathie $(2,7)$ ist neben einer anzunehmenden Funktionseinschränkung der Schleimhaut nach unseren Befunden auch durch Reaktion mit Leukocytenproteasen $\mathrm{zu}$ erklären. Solange die Leukocytenproteasen durch genügende Inhibitorproduktion gehemmt werden können, treten in den Nasensekreten auch keine anderen Proteasen auf. Diese unspezifischen, wahrscheinlich bakteriellen Proteasen treten erst im akuten oder chronischen Stadium von Rhinopathien auf, offensichtlich erst nach Erschöpfung des inhibierenden Systems für leukocytäre Proteasen. Die Reinigung der leukocytären Proteasen und ihre Hemmbarkeit durch Sekretinhibitor sind Gegenstand weiterer Untersuchungen.

Das Fehlen der Hemmwirkung des Inhibitors gegen Plasmin erscheint physiologisch sinnvoll, da ein so hoher Hemmstoffgehalt eine Thrombosierung der Nasenschleimhaut verursachen könnte. Ebenso ist das Fehlen der Hemmwirkung gegen das vasodilatorisch wirkende Kallikrein-Kininsystem von großer Bedeutung. Die Funktionsfähigkeit des Systems ist auch unter physiologischen Bedingungen, d. h. bei hohem Inhibitorspiegel, gewährleistet.
Die Hemmwirkung des Sekretinhibitors gegen Leukocytenproteasen erscheint besonders bemerkenswert, wenn man die ähnliche Wirkung des $\alpha_{1}$-Antitrypsins des Serums auf Leukocytenproteasen in Betracht zieht. Bei Ausfall dieses antiproteolytischen Systems, z. B. bei Gendefekten, sind in vielen Fällen Lungenemphyseme zu beobachten. Dem $\alpha_{1}$-Antitrypsin wird daher in den Lungenalveolen eine primär nicht gegen die Keime gerichtete Schutzfunktion bei Infektionen zugeschrieben. Diese Schutzfunktion ist möglicherweise auf die inhibierende Wirkung gegen freiwerdende Leukocytenproteasen zurückzuführen. Aus diesen Beobachtungen kann abgeleitet werden, daß eine solche Schutzfunktion offensichtlich im oberen Respirationstrakt der von uns beschriebene Inhibitor übernimmt. Dies erscheint sinnvoll, da $\alpha_{1}$-Antitrypsin mit einem Molekulargewicht von 45000 die Membranen offenbar nur schwer durchdringen kann, während der spezifische Sekretinhibitor mit einem Molekulargewicht von etwa 13000 wesentlich leichter sezerniert werden kann.

Trotz der hohen antiproteolytischen Wirkung des Blutplasmas erfordert die „Blut-Sekretschranke“ jeweils die Produktion eines besonderen Inhibitors (8). So wird auch der Schutz des Pankreas vor Autolyse durch aktivierte Proteasen nicht durch $\alpha_{1}$-Antitrypsin sondern durch einen spezifischen, niedermolekularen Inhibitor gewährleistet.

Die Frage, ob der von uns untersuchte Inhibitor mit einem Trypsin-Chymotrypsin-Inhibitor der Tränenflüssigkeit (9) identisch ist, kann erst nach Reindarstellung beider Inhibitoren entschieden werden. Wir nehmen aber an, daß es sich in unserem Fall um einen spezifischen Inhibitor handelt, da wir ihn in allen Sekreten des Flimmerepithels und in allen mit Flimmerepithel ausgekleideten Schleimhäuten des oberen Respirationstraktes nachweisen können.

\section{Literatur}

1. Hochstrasser, K., H. Haendle, R. Reichert und E. Werle, Hoppe-Seyler's Z. physiol. Chem. 352, 954 (1971). - 2. REICIIERT, R., K. Hochstrasser und E. WerLe, Klin. Wschr. 49, 1234 (1971). 3. FrEY, E. K., H. KraUt und E. WERLE, Das Kallikrein-Kininsystem und seine Inhibitoren, Ferd. Enke Verlag, Stuttgart (1968). 4. Fritz, H., E. Jaumani, R. Meister, P. Pasquay, K. Hochstrasser und E. Fink, Proc. of the Int. Res. Conf. on Proteinase Inhibitors, Walter de Gruyter Verlag (1971). - 5. HOCHSTRASSER, K., K. Illchmann und E. Werle, Hoppe-Seyler's Z. physiol.
Chem. 350, 929 (1969). - 6. Nagel, W., F. Willig, W. Peschke und F. H. Scrimidr, Hoppe-Seyler's Z. physiol. Chem. 340, 1 (1961). - 7. ReIChert, R. und K. Hochstrasser, in Vorbereitung. 8. Voger, R., I. Trautschold und E. Werle, Natural Proteinase Inhibitors, Academic Press, New York-London (1968). - 9. Küppers, F., Biochim. biophysica Acta, Amsterdam 229, 845 (1971). - 10. BERGMEYER, H. U., Methoden der enzymatischen Analyse, 2. Auf. Bd. I. S. 1029, Verlag Chemie Weinheim/Bergstr. (1970)
Priv.-Doz. Dr. K. Hochstraßer Dr. R. Reichert HNO-Klinik der Universität 8 München 15 Pettenkoferstr. 4a 